\title{
Effect of Group Delay Dispersion on Femtosecond Pulse Self-shortening in Kerr Media: Investigation of Femtosecond Pulse Self-shortening Phenomenon Based on the Transient Regime of Multiple Filamentation in Kerr Media with Different Group Delay Dispersion
}

\author{
Andrew V. Koribut', Yakov V. Grudtsin², and Leonid D. Mikheev²,3 \\ ${ }^{1}$ Moscow Institute of Physics and Technology (State University), Dolgoprudny, Moscow Region, \\ Russia \\ ${ }^{2}$ P. N. Lebedev Physical Institute, Moscow, Russia \\ ${ }^{3}$ National Research Nuclear University MEPhl (Moscow Engineering Physics Institute), \\ Kashirskoe shosse 31, Moscow, 115409, Russia
}

Corresponding Author: Yakov V. Grudtsin jgrudtsin@gmail.com

Received: 22 July 2018 Accepted: 9 September 2018 Published: 8 October 2018

Publishing services provided by Knowledge $\mathrm{E}$

(c) Andrew V. Koribut et al. This article is distributed under the terms of the Creative Commons Attribution License, which permits unrestricted use and redistribution provided that the original author and source are credited.

Selection and Peer-review under the responsibility of the Breakthrough Directions of Scientific Research at MEPhl Conference Committee.

\section{Abstract}

We present the results of the investigation of the effect of material dispersion on a novel femtosecond pulse self-shortening mechanism based on the transient regime of multiple filamentation in samples with Kerr nonlinearity. This regime occurs at rather high B-integral values for the pulse passing through the sample, so that multiple filamentation in the trailing part causes strong on-axis intensity losses due to diffraction and refraction that allows far-field spatial filtering of the pulse front edge unaffected by the multiple filamentation. Self-shortening of a 72 fs transform-limited pulse to $23 \mathrm{fs}, 32 \mathrm{fs}$ and $30 \mathrm{fs}$ was observed in a 1-mm-thick fused silica plate at intensity of $2.9 \mathrm{TW} / \mathrm{cm}^{2}$, a 3-mm-thick fused silica plate at intensity of $1.1 \mathrm{TW} / \mathrm{cm}^{2}$ and a 5 -mm-thick LiF plate at intensity of $2.1 \mathrm{TW} / \mathrm{cm}^{2}$, respectively, without the use of additional dispersive elements. Experimental results obtained in this article show that the duration of self-shorted pulses increases with increasing group delay dispersion in the material.

Keywords: multiple filamentation, pulse self-shortening

\section{Introduction}

To date, various fields of basic and applied research require few-cycle optical pulses [1-3]. However, it is difficult to obtain pulses shorter than 20-30 fs in multi-terawatt and petawatt power level solid state systems because of spectrum narrowing during 
pulse amplification. The solution to this problem lies in the development of nonlinear methods for the extracavity shortening of femtosecond pulses [4, 5]. The methods currently used are based on the spectrum broadening due to self-phase modulation (SPM) in media with Kerr nonlinearity with compensation of the acquired phase modulation by using additional dispersive elements or without the use of these elements if the transform-limited pulses are formed directly in a nonlinear medium (selfcompression). At the same time, currently developed self-compression methods are limited in energy by tens of $\mathrm{m}$ and do not have the prospect of further energy scaling $[6,7]$. Earlier, we presented the results of investigations of a new mechanism for femtosecond pulse self-shortening in thin optically transparent materials with Kerr nonlinearity [8-11]. In [11], this mechanism was attributed to the formation of multiple filaments in the trailing edge of the pulse, which leads to a sharp increase in diffraction and refraction losses in the near-axis region. The mechanism of the observed phenomenon allows energy scaling of the self-shortening technique. In this article, we study effect of the group delay dispersion on femtosecond pulse self-shortening in Kerr media.

\section{Experimental Setup and Results}

The scheme of the experimental setup is shown in Figure 1. The duration of the initial transform-limited $200 \mu$ pulse was $72 \mathrm{fs}$ at a wavelength of $475 \mathrm{~nm}$. Experiments were carried out with three samples: 1-mm and 3-mm-thick silica plates and a 5-mm-thick LiF plate. The radiation intensity on samples varied in the range of $1-2.9 \mathrm{TW} / \mathrm{cm}^{2}$ by moving them along a converging beam. Using $60 \mu \mathrm{m}$ or $100 \mu \mathrm{m}$ pinholes, adjacent to the back surface of the sample, a region of uniform intensity in the central part of the beam was selected. The radiation at the exit of the pinholes consisted of a central core, whose size did not depend on the interaction intensity, and conical emission with a divergence angle of up to $0.1 \mathrm{rad}$ (the angle of convergence of the initial radiation was $<0.01$ rad).

In the 1-mm-thick fused silica plate, the radiation spectrum observed at intensity of $2.9 \mathrm{TW} / \mathrm{cm}^{2}$ was broadened and redshifted to a wavelength of $\sim 490 \mathrm{~nm}$ with the pulse duration being reduced to $22 \mathrm{fs}$ (Figure 2a). A similar phenomenon of the pulse self-shortening was observed in the 3-mm-thick silica plate at an intensity of $1.1 \mathrm{TW} / \mathrm{cm}^{2}$ (Figure $2 \mathrm{~b}$ ) and in the 5 -mm-thick LiF sample at an intensity of $2.1 \mathrm{TW} / \mathrm{cm}^{2}$ (Figure 2c). 

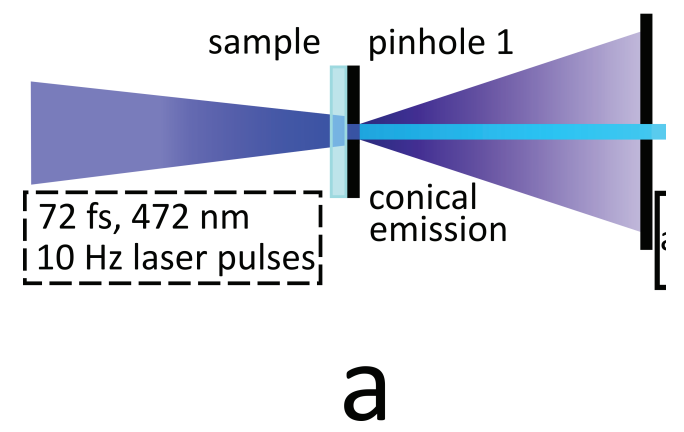

Figure 1: Experimental setup for the observation of pulse self-shortening (a) and fluence distribution for the beam at a distance of $5 \mathrm{~cm}$ from the pinhole $1(\mathrm{~b})$.
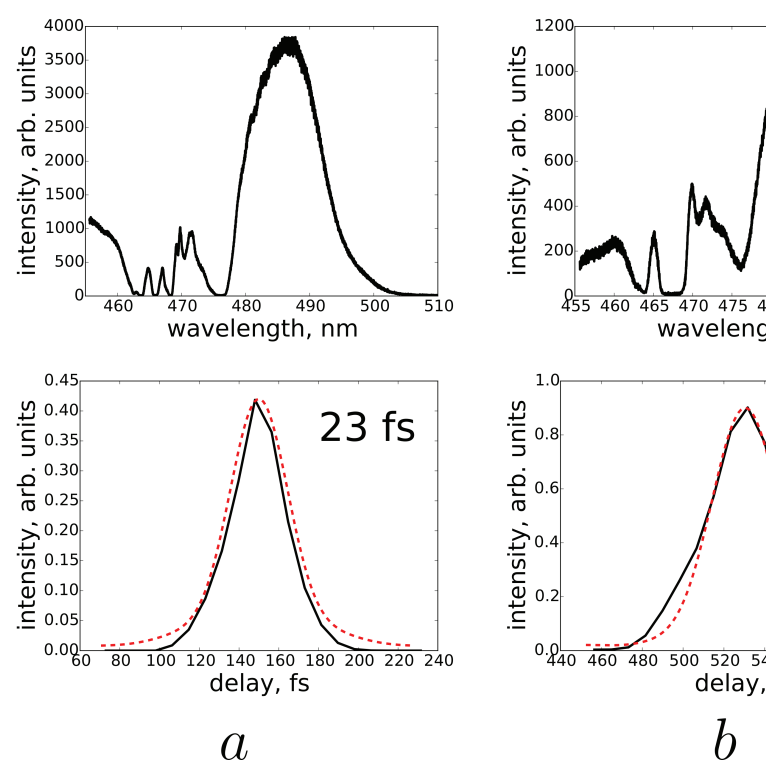

Figure 2: On-axis spectra and autocorrelation traces of pulses passed through: (a) 1-mm-thick fused silica plate, (b) 3-mm-thick fused silica plate, (c) 5-mm-thick LiF plate. Dashed curves: autocorrelation functions reconstructed from the inverse Fourier transform of the measured spectra.

\section{Discussion}

According to [11], self-shortening process is caused by the multiple filamentation in the trailing part that arises due to the transverse modulational instability [12], which leads to the strong on-axis intensity losses due to diffraction and refraction. At the same time, radiation from the leading edge of the pulse is not affected by the multiple filamentation and keeps its divergence close to the initial value. SPM enriches and red-shifts the spectrum in the leading part of the pulse.

Spatial fluctuations of the beam intensity grow during pulse propagation with the gain determined by the B-integral, which can be roughly estimated as $\frac{2 \pi}{\lambda} n_{2} I L$, where $\mathrm{n}_{2}$ is the nonlinear refractive index; l, radiation intensity, and L, sample thickness. Using $\mathrm{n}_{2}=2.2 \times 10^{-16} \mathrm{~cm}^{2} / \mathrm{W}$ for fused silica and $\mathrm{n}_{2}=5.3 \times 10^{-17} \mathrm{~cm}^{2} / \mathrm{W}$ for LiF [13], we can obtain 


$$
\begin{aligned}
& B_{1}\left(2.9 \mathrm{TW} / \mathrm{cm}^{2}, 1 \mathrm{~mm} \text {, fused silica }\right)=8.5 \\
& B_{2}\left(1.1 \mathrm{TW} / \mathrm{cm}^{2}, 3 \mathrm{~mm} \text {, fused silica }\right)=9.4 \\
& B_{3}\left(2.1 \mathrm{TW} / \mathrm{cm}^{2}, 5 \mathrm{~mm}, \mathrm{LiF}\right)=7.4
\end{aligned}
$$

Despite the fact that the values of the B-integral are close to each other, the output pulses have different durations, which is most clearly seen from comparing the 1 - and 3-mm-thick fused silica samples. However, these samples have different group delay dispersion (GDD), namely $75 \mathrm{fs}^{2}$ and $225 \mathrm{fs}^{2}$ for the 1 - and 3-mm-thick fused silica samples [14], respectively, as well as $210 \mathrm{fs}^{2}$ for LiF [15]. GDD results in temporal pulse broadening, which reduces the real value of the B-integral and increases the duration of the leading edge. As a result, this leads to an increase in the duration of the selfshorted pulse with sample thickness (Figure 2). The differences in the real values of the B-integral explain the different spectrum broadening and red-shifting. Despite the different group delay dispersion, the self-shorted pulses are close to the transformlimited ones.

Since the GDD depends on the sample thickness and the group velocity dispersion (GVD), to maintain the interaction regime (B-integral value) to be optimal for the selfcompression, it is necessary to choose a material with a large nonlinear refractive index $\mathrm{n}_{2}$ and a small GVD. A CaF ${ }_{2}$ sample could be promising for self-shortening $\left(\mathrm{n}_{2}=\right.$ $4 \times 10^{-16} \mathrm{~cm}^{2} / \mathrm{W}$ [16], GVD $=53 \mathrm{fs}^{2} / \mathrm{mm}$ [17] at $\left.\lambda=480 \mathrm{~nm}\right)$. For near infrared region, the dispersion factor can be further reduced (for $\mathrm{LiCl} \mathrm{n}_{2}=4 \times 10^{-16} \mathrm{~cm}^{2} / \mathrm{W}$ [18], GVD = $10 \mathrm{fs}^{2} / \mathrm{mm}[15]$ at $\left.\lambda=1064 \mathrm{~nm}\right)$.

\section{Summary}

We have observed pulse self-shortening phenomenon based on the transient regime of multiple filamentation in samples with different GDD. The experimentally obtained results demonstrate that to generate pulses with shorter duration, samples with small dispersion factor should be used.

\section{Acknowledgment}

This work was supported by the Presidium of the Russian Academy of Sciences (Programme 'Extreme laser radiation: physics and fundamental applications'), and the Competitiveness Programme of the National Nuclear Research University MEPhl (Moscow Engineering Physics Institute). 


\section{References}

[1] Kobayashi, T., Shirakawa, A., and Fuji, T. (2001). Sub-5-fs transform-limited visible pulse source and its application to real-time spectroscopy. IEEE Journal of Selected Topics in Quantum Electronics, vol. 7, no. 4, pp. 525-538.

[2] Zhou, M. L., Yan, X. Q., Mourou, G., et al. (2016). Proton acceleration by single-cycle laser pulses offers a novel monoenergetic and stable operating regime. Physics of Plasmas, vol. 23, no. 4, p. 043112.

[3] Witte, S., Zinkstok, R. T., Hogervorst, W., et al. (2005). Generation of few-cycle terawatt light pulses using optical parametric chirped pulse amplification. Optics Express, vol. 13, no. 13, pp. 4903-4908.

[4] Rolland, C. and Corkum, P. B. (1988). Compression of high-power optical pulses. JOSA $B$, vol. 5, no. 3, pp. 641-647.

[5] Nisoli, M., De Silvestri, S., and Svelto, O. (1996). Generation of high energy 10 fs pulses by a new pulse compression technique. Applied Physics Letters, vol. 68, no. 20, pp. 2793-2795.

[6] Stibenz, G., Zhavoronkov, N., and Steinmeyer, G. (2006). Self-compression of millijoule pulses to $7.8 \mathrm{fs}$ duration in a white-light filament. Optics letters, vol. 31, no. 2 , pp. $274-276$.

[7] Ashihara, S., Nishina, J., Shimura, T., et al. (2002). Soliton compression of femtosecond pulses in quadratic media. JOSA B, vol. 19, no. 10, pp. 2505-2510.

[8] Aristov, A. I., Grudtsyn, Ya. V., Mikheev, L. D., et al. (2012). Spectral broadening and self-compression of negatively chirped visible femtosecond pulses in fused silica. Quantum Electronics, vol. 42, no. 12, pp. 1097-1099.

[9] Alekseev, S. V., Aristov, A. I., Grudtsyn, Ya. V., et al. (2013). Visible-range hybrid femtosecond systems based on a XeF (C-A) amplifier: State of the art and prospects. Quantum Electronics, vol. 43, no. 3, p. 190.

[10] Grudtsyn, Y. V., Zubarev, I. G. E., Koribut, A. V., et al. (2015). Self-phase modulation in a thin fused silica plate upon interaction with a converging beam of down-chirped femtosecond radiation. Quantum Electronics, vol. 45, no. 5, p. 415.

[11] Grudtsyn, Ya. V., Koribut, A. V., Trofimov, V. A., et al. (unpublished). Jellyfish-like mechanism of femtosecond pulse self-shortening in Kerr media. JOSA B.

[12] Bespalov, V. I. and Talanov, V. I. (1966). Filamentary structure of light beams in nonlinear liquids. ZhETF Pisma Redaktsiiu, vol. 3, p. 471. 
[13] DeSalvo, R., Said, A. A., Hagan, D. J., et al. (1996). Infrared to ultraviolet measurements of two-photon absorption and $\mathrm{n}_{2}$ in wide bandgap solids. IEEE Journal of Quantum Electronics, vol. 32, no. 8, pp. 1324-1333.

[14] Malitson, I. H. (1965). Interspecimen comparison of the refractive index of fused silica. Josa, vol. 55, no. 10, pp. 1205-1209.

[15] Li, H. H. (1976). Refractive index of alkali halides and its wavelength and temperature derivatives. Journal of physical and chemical reference data, vol. 5, no. 2, pp. 329-528.

[16] Levenson, M. (1974). Feasibility of measuring the nonlinear index of refraction by third-order frequency mixing. IEEE Journal of Quantum Electronics, vol. 10, no. 2, pp. 110-115.

[17] Malitson, I. H. (1963). A redetermination of some optical properties of calcium fluoride. Applied Optics, vol. 2, no. 11, pp. 1103-1107.

[18] Penzkofer, A., Schmailzl, J., and Glas, H. (1982). Four-wave mixing in alkali halide crystals and aqueous solutions. Applied Physics B: Lasers and Optics, vol. 29, no. 1, pp. 37-42. 